\title{
BMJ Open Pre-Adolescent Cardio-Metabolic Associations and Correlates: PACMAC methodology and study protocol
}

\author{
Nicholas Castro, ${ }^{1}$ James Faulkner, ${ }^{1}$ Paula Skidmore, ${ }^{2}$ Michelle Williams, ${ }^{3}$ \\ Danielle M Lambrick, ${ }^{4}$ Leigh Signal, ${ }^{5}$ Michelle Thunders, ${ }^{4}$ Diane Muller, ${ }^{5}$ \\ Sally Lark, ${ }^{1}$ Mike Hamlin, ${ }^{6}$ Andrew M Lane, ${ }^{7}$ Te Kani Kingi, ${ }^{8}$ Lee Stoner ${ }^{1}$
}

To cite: Castro N, Faulkner J, Skidmore $\mathrm{P}$, et al. Pre-Adolescent CardioMetabolic Associations and Correlates: PACMAC methodology and study protocol. BMJ Open 2014:4: e005815. doi:10.1136/ bmjopen-2014-005815

- Prepublication history for this paper is available online. To view these files please visit the journal online (http://dx.doi.org/10.1136/ bmjopen-2014-005815)

Received 9 June 2014 Revised 6 August 2014 Accepted 27 August 2014

CrossMark

For numbered affiliations see end of article.

Correspondence to Nicholas Castro;

N.Castro@massey.ac.nz

\section{ABSTRACT}

Introduction: Although cardiovascular disease is typically associated with middle or old age, the atherosclerotic process often initiates early in childhood. The process of atherosclerosis appears to be occurring at an increasing rate, even in pre-adolescents, and has been linked to the childhood obesity epidemic. This study will investigate the relationships between obesity, lifestyle behaviours and cardiometabolic health in prepubescent children aged 8-10 years, and investigates whether there are differences in the correlates of cardiometabolic health between Māori and Caucasian children. Details of the methodological aspects of recruitment, inclusion/exclusion criteria, assessments, statistical analyses, dissemination of findings and anticipated impact are described.

Methods and analysis: Phase 1: a cross-sectional study design will be used to investigate relationships between obesity, lifestyle behaviours (nutrition, physical activity/fitness, sleep behaviour, psychosocial influences) and cardiometabolic health in a sample of 400 pre-pubescent (8-10 years old) children. Phase 2: in a subgroup (50 Caucasian, 50 Māori children), additional measurements of cardiometabolic health and lifestyle behaviours will be obtained to provide objective and detailed data. General linear models and logistic regression will be used to investigate the strongest correlate of (1) fatness; (2) physical activity; (3) nutritional behaviours and (4) cardiometabolic health.

Ethics and dissemination: Ethical approval will be obtained from the New Zealand Health and Disabilities Ethics Committee. The findings from this study will elucidate targets for decreasing obesity and improving cardiometabolic health among preadolescent children in New Zealand. The aim is to ensure an immediate impact by disseminating these findings in an applicable manner via popular media and traditional academic forums. Most importantly, results from the study will be disseminated to participating schools and relevant Māori health entities.

\section{INTRODUCTION}

While cardiovascular disease (CVD) is typically associated with middle or old age, the

\section{Strengths and limitations of this study}

- Worldwide, health systems are struggling under the escalating burden of non-communicable diseases.

- Increasing rates of cardiometabolic complications among preadolescents have been linked to the childhood obesity epidemic.

- This study will comprehensively examine the relationships between obesity, lifestyle behaviours and cardiometabolic health in preadolescents.

- The stated research will increase the understanding of current diseases affecting New Zealand children.

- Findings from this study will enlighten targets for improving cardiometabolic health among preadolescents in New Zealand.

atherosclerotic process often initiates early in childhood. ${ }^{1}$ The process of atherosclerosis appears to be occurring at an increasing rate, even in preadolescents, and has been linked to the childhood obesity epidemic. ${ }^{2} 3$ In New Zealand, the prevalence of childhood (2-14 years) obesity increased from $8.4 \%$ in $2006 / 2007$ to $11.1 \%$ in $2012 / 2013$. Furthermore, within New Zealand, much higher rates of obesity have been reported among Māori children (18.6\%) than among their counterparts of European ancestry $(7.5 \%) .{ }^{4}$ The prevalence of obesity has taken its toll among the Māori population, placing these cohorts at greater risk for obesity-related cardiometabolic complications, including dyslipidemia, hypertension, type 2 diabetes mellitus and subsequent CVD. ${ }^{5}$

A child's body composition is determined by interactions between their genes and lifestyle behaviours. The genetic makeup of children has not altered dramatically over the past two decades in which obesity has rapidly increased. It is likely, therefore, that lifestyle behaviours and environmental influences are a probable cause of this childhood obesity 
epidemic. ${ }^{5}{ }^{6}$ While commonly cited causes of obesity include declining physical activity and fitness levels, ${ }^{7}$ poor nutrition ${ }^{7}$ and poor sleep habits, ${ }^{8}$ no study in New Zealand has comprehensively assessed all of these components together in primary school-aged children. Furthermore, it is unknown whether (A) fatness (body composition), physical fitness, physical activity, nutritional behaviour or sleep is the most important correlate (strongest independent predictor) of a child's cardiometabolic health and (B) whether these relationships are consistent between Māori and Caucasian children. The findings from this study may allow us to develop culturally appropriate interventions for children to promote sustainable obesity-preventing lifestyles and to improve cardiovascular health. ${ }^{2}$

Our aim is to investigate the relationships between obesity, lifestyle behaviours (nutrition, physical activity/ fitness, sleep behaviour, psychosocial influences) and cardiometabolic health in pre-pubescent $(8-10$ years old) children. Furthermore, the research will investigate whether there are differences in the correlates of cardiometabolic health between Māori and Caucasian children. The research outcomes from Pre-Adolescent CardioMetabolic Associations and Correlates (PACMAC) will help inform health professionals, health and physical education curricular and child health policy guidelines as to the most effective way to make an impact on the deteriorating health of children. The stated research will increase the understanding of CVDs that are affecting New Zealanders, and subsequently through more targeted interventions enhance the health and well-being of children and consequently adults.

General Research Questions: Phase 1

1. Investigate the associations of physical activity, physical fitness, nutrition and sleep habits with fatness.

2. Investigate the strongest correlate of physical activity: physical environment (walkability, playability index) or psychosocial (attraction to physical activity).

3. Investigate the strongest correlate of nutritional behaviour: physical environment (local built food environment), psychosocial (preferences for and attitudes towards healthy foods) or hereditary (genetic variability of taste receptors).

4. Investigate the association of fatness, nutrition, physical activity, physical fitness and sleep with cardiometabolic health.

Figure 1 Protocol for phase 1 (20-MST, 20 m Multistage Shuttle Run test; BIA, bio-impedance analysis; BMI, body mass index; PWA, pulse wave analysis; WHR, waist:hip ratio).
General Research Questions: Phase 2

5. Using objective measures (A) investigate the association of fatness, nutrition, physical activity, physical fitness and sleep with CVD risk and (B) determine whether the strongest correlate of CVD risk is similar for Māori and Caucasian children.

\section{METHODS}

\section{Study design}

A cross-sectional study design will be used to investigate relationships between obesity, lifestyle behaviours and cardiometabolic health in a sample of pre-pubescent children aged 8-10 years from three representative sample sites from across New Zealand (Wellington, Canterbury and Otago). Data collection will begin in October 2014 and will continue for 18 months. Phase 1: To reduce disruption and burden for the children, previously validated broadly applicable research instruments, which have been used in large-scale national and multinational epidemiological studies, will be utilised (see figure 1). Phase 2: In a subgroup of children (50 Māori, 50 Caucasian children) objective measurements will be obtained to provide additional more in-depth data to add to our findings (see figure 2).

\section{Study participants and recruitment}

Four hundred children will be recruited for participation in phase 1 where measures will be largely based on self-report, of which 100 children will also be asked to participate in phase 2 (objective). In addition to having self-reported measures, objective measures will be taken. Children will be deemed eligible if they are aged 810 years and are asymptomatic of injury or illness as determined by a standardised health screening questionnaire and a physical activity readiness-questionnaire (PAR-Q), completed by a parent or guardian. Children will be ineligible to participate if they have had an orthopaedic injury or surgery that has prohibited full function within the previous 4 weeks or are prescribed any cardiovascular medications. Written parental or guardian consent and child assent will be obtained prior to participation.

Schools (Wellington, Canterbury and Otago) will be stratified by socioeconomic status and randomly selected. School principals will be contacted initially via 
Figure 2 Protocol for phase 2 (BIA, bio-impedance analysis; BMI, body mass index; FMD, flow-mediated dilation; HRV, heart rate variability; $P A$, physical activity (accelerometry); PWA, pulse wave analysis; WHR, waist: hip ratio; $\mathrm{VO}_{2}$ max, maximal oxygen uptake.

\begin{tabular}{|c|c|c|c|c|c|c|c|c|}
\hline Day & \multicolumn{6}{|c|}{1} & $1-7$ & 7 \\
\hline Location & \multicolumn{6}{|c|}{ Laboratory } & & Home \\
\hline Outcome & $\begin{array}{l}\text { BMI } \\
\text { WHR } \\
\text { BIA }\end{array}$ & $\begin{array}{l}\text { Taste } \\
\text { DNA }\end{array}$ & Rest & $\begin{array}{c}\text { Visceral } \\
\text { Fat }\end{array}$ & $\begin{array}{l}\text { FMD } \\
\text { HRV } \\
\text { PWA }\end{array}$ & $\mathrm{VO}_{2} \max$ & $\begin{array}{c}\text { Sleep } \\
\text { PA }\end{array}$ & $\begin{array}{l}\text { Web } \\
\text { Survey }\end{array}$ \\
\hline Mins: & 5 & 5 & 15 & 5 & 25 & 10 & 10 & 60 \\
\hline
\end{tabular}

email invitation, followed by a face-to-face meeting. Written consent will be sought from both the principal and the classroom teachers of each school before participants in year 3-5 classes are recruited. Subsequently, an information packet will be sent home with the children, and the children will be eligible to participate if they return the parent informed consent and child assent forms signed.

\section{Cultural sensitivity}

A number of steps will be included within the design of the study to ensure cultural sensitivity and long-term viability of PACMAC:

1. Māori academic consultants have been involved in the development of the study and will have an ongoing role in advising on cultural issues to ensure that the study findings are appropriately delivered to Māori communities.

2. Guidance will also be provided on acceptable methods of engaging Māori participants, on cultural issues that may arise in relation to the study design and measures and how these might inform the development and success of the study.

\section{Phase 1 procedures (400 children)}

The outline for phase 1 is depicted in figure 1. Monday through Thursday, anthropometric, cardiometabolic and taste outcomes will be assessed. Students will report for testing between the hours of 9:00 and 12:00, having fasted for at least $3 \mathrm{~h}$, and refrained from exercise for $24 \mathrm{~h}^{9}$ Assessments (table 1) will take place in a quiet, climate controlled room at the school. On Friday, all children will have their physical fitness assessed in the school hall. A web-based (Lime Survey, open source) questionnaire will be used to collect demographic data, as well as psychosocial, sleep behaviour, physical activity and nutrition behaviour using previously validated scales. The questionnaire will be web based, and will be completed at the child's home, in consultation with a parent or guardian.

\section{Demographic}

Demographic data will include home and school address, gender, ethnicity, parent marital status, parent occupation, parent academic qualifications and household income. Home address will be used to determine urban versus rural residential area and New Zealand deprivation score used as a measure of neighbourhood socioeconomic status. The school address will be used to calculate school decile rating.

Table 1 Study outcomes

\begin{tabular}{|c|c|c|}
\hline Methods & Phase 1 & Phase 2 \\
\hline \multicolumn{3}{|l|}{ Cardiometabolic } \\
\hline Flow-mediated dilation & & $x$ \\
\hline Heart rate variability & & $x$ \\
\hline Pulse wave analysis & $\mathrm{x}$ & $x$ \\
\hline $\begin{array}{l}\text { Blood markers: lipoproteins, } \\
\text { triglycerides, serum glucose, } \\
\text { HbA1c }\end{array}$ & $x$ & \\
\hline \multicolumn{3}{|l|}{ Fatness } \\
\hline Visceral fat & & $x$ \\
\hline Waist:hip ratio, waist:height ratio & $x$ & $x$ \\
\hline Body mass index & $x$ & $x$ \\
\hline Bio-impedance analysis & $x$ & $x$ \\
\hline \multicolumn{3}{|l|}{ Physical activity } \\
\hline Accelerometry & & $x$ \\
\hline $\begin{array}{l}\text { Youth Physical Activity } \\
\text { Questionnaire }\end{array}$ & $\mathrm{x}$ & $x$ \\
\hline $\begin{array}{l}\text { Children's Attraction to Physical } \\
\text { Activity scale }\end{array}$ & $x$ & $x$ \\
\hline $\begin{array}{l}\text { Neighbourhood/school walkability, } \\
\text { playability index }\end{array}$ & $x$ & $\mathrm{x}$ \\
\hline \multicolumn{3}{|l|}{ Physical fitness } \\
\hline Cycle $\mathrm{VO}_{2} \max$ & & $x$ \\
\hline Shuttle run test (20-MST) & $x$ & \\
\hline \multicolumn{3}{|l|}{ Sleep } \\
\hline Accelerometry (+sleep diary) & & $x$ \\
\hline Child Sleep Habits Questionnaire & $x$ & $x$ \\
\hline \multicolumn{3}{|l|}{ Nutrition } \\
\hline $\begin{array}{l}\text { New Zealand Children's Food } \\
\text { Frequency Questionnaire }\end{array}$ & $x$ & $x$ \\
\hline $\begin{array}{l}\text { Psycho-social Correlates of Food } \\
\text { Choice Questionnaire }\end{array}$ & $\mathrm{x}$ & $x$ \\
\hline Four-day estimated food diary & & $x$ \\
\hline Food outlet availability & $\mathrm{x}$ & $x$ \\
\hline Taste test & $x$ & $x$ \\
\hline DNA (saliva) & $x$ & $x$ \\
\hline
\end{tabular}

20-MST, 20 m Multistage Shuttle Run test; HbA1c, glycated haemoglobin; $\mathrm{VO}_{2} \mathrm{max}$, maximal oxygen uptake. 


\section{Cardiometabolic health}

Biochemical markers (total cholesterol, high-density lipoproteins, low-density lipoproteins, triglycerides, serum glucose and glycated haemoglobin (HbAlc)) will be supplemented with pulse wave analysis (PWA). Biochemical markers will be collected using a welltolerated finger prick procedure and analysed using portable lipid/glucose (CardioChek PA) and HbAlc (Afinion HbA1c) analysers. PWA will estimate central systolic blood pressure (cSBP) and central arterial wave reflection (augmentation index, AIx \%). PWA is a simple, non-invasive, valid and reliable technique that has been widely used in epidemiological studies. ${ }^{9-11}$ Each variable will be standardised as follows: standardised value $=($ value - mean $) / S D$. The $z$-scores of the individual risk factors will be summed to create a cardiometabolic risk score.

\section{Fatness}

Body composition will be estimated using body mass index (BMI), waist circumference, waist:hip ratio (WHR), waist:height ratio and body composition (fat mass, fat-free mass) using bioimpedance analysis. Fat mass index (FMI) will be calculated by dividing fat mass $(\mathrm{kg})$ by height squared (m) and fat-free mass index (FFMI) by dividing fat-free mass $(\mathrm{kg})$ by height squared $(\mathrm{m})$. Using BMI, children will be classified as normal weight, overweight or obese according to International Obesity Task Force cut-off points. ${ }^{12}$

\section{Physical fitness}

Cardiorespiratory fitness will be estimated using the $20 \mathrm{~m}$ maximal multistage shuttle run test (20-MST), a reliable and valid test which requires limited facilities. ${ }^{13}$ The results are recorded as laps taken until exhaustion.

\section{Physical activity}

Moderate-to-vigorous physical activity (MVPA) $\mathrm{min} /$ day, as well as physical activity type and context (eg, modality), will be determined using the Youth Physical Activity Questionnaire (YPAQ). The 47-item YPAQ asks students to quantify the frequency and duration of a range of physical and sedentary activities for both week and weekend days over the past 7 days.

\section{Nutrition}

Food choice will be assessed using a specially modified, short version of New Zealand Adolescent Food Frequency Questionnaire (NZAFFQ), the New Zealand Children's Food Frequency Questionnaire (NZCFFQ), designed for use in this age group. This Food Frequency Questionnaire (FFQ) is based on the Health Behaviour in School Children (HBSC) FFQ, which is suitable for use in this age group. This questionnaire was pretested in a sample of adolescents from Otago before use in a study and showed good repeatability and relative validity. ${ }^{14}$ This is a nonquantitative FFQ (ie, it measures frequency only, not portion size). Frequency of consumption of 15 commonly consumed food items will be recorded by asking the respondent how many times weekly each item is consumed. Dietary patterns will be generated from this FFQ data using principal components analysis.

\section{Sleep}

Increasing evidence has indicated that short sleep duration may be related to cardiometabolic complications, including obesity, ${ }^{15}$ among children. Sleep habits and sleep disturbances will be recorded using the Children's Sleep Habits Questionnaire (CSHQ). ${ }^{16}$ A total score and scores on the eight subscales that cover key domains of sleep and sleep behaviour (bedtime resistance, sleep onset, sleep duration, anxiety around sleep, night waking, parasomnias, sleep-disordered breathing and morning waking/daytime sleepiness) will be calculated.

\section{Environmental correlates of physical activity and nutritional behaviour}

Student's home and school locations will be mapped using a Geographic Information System (GIS) package (ArcGIS 10, ESRI). Two physical activity-related variables (neighbourhood walkability index, playability index) and several food outlet accessibility variables will be calculated. Personalised walkability index values will be calculated using combined measures of street connectivity, dwelling density, land use mix and retail floor area ratio, as previously reported by URBAN Study. ${ }^{17}$ The buffer distance will be developed along an $880 \mathrm{~m}$ (corresponding to 10 min walk) street network from the student's residence, excluding areas that cannot be accessed due to major barriers (eg, roads, water features). Playability index values will be calculated based on public park proximity/availability and quality features within them presumed to be most related to physical activity among children aged $8-10$ years. ${ }^{18}$

Personalised food environment index will be calculated by determining the density of 'healthy' and "'nonhealthy' outlets, as well as individual types of outlets (all food outlets, convenience stores, supermarkets, fast food outlets) along an $880 \mathrm{~m}$ street network from the student's residence, and distance to the nearest outlet. Unhealthy food outlets were those that offered primarily energy dense, low-nutrient foods, such as chain fast food restaurants. This index will be categorised using a median split to determine if the food environment is 'healthy' $(<50$ th centile of unhealthy food outlets/ healthy food outlets) or 'unhealthy' ( $\geq 50$ th centile of unhealthy food outlets/healthy food outlets).

\section{Psychosocial determinants of physical activity and nutritional behaviour}

The Children's Attraction to Physical Activity scale $(\mathrm{CAPA})^{19}$ includes 25 items and 5 dimensions: (1) enjoyment of vigorous physical activity, (2) perceived importance of participating in physical activity, (3) liking of games and sports, (4) perception of physical exertion as fun and (5) perceived peer acceptance in games and 
sports. The modified version of CAPA, using a Likert format, has been validated for use with young (aged 68 years) children. ${ }^{20}$ Each subscale includes five items, measured from one (low) to four (high). An average score (of four) will be calculated for each subscale.

Psychosocial correlates of food choice will be assessed using the Pro Children study questionnaire, ${ }^{21}$ which includes questions on the importance of healthy eating, attitudes and beliefs towards healthy eating, self-efficacy and home availability. Responses these questions will be summed to provide scores for each domain.

\section{Taste}

Eating behaviours are influenced by taste preference and access to foods in the environment. Food outlet accessibility will be calculated as described above. Humans exhibit considerable variation in their taste and olfactory receptors, ${ }^{22}$ which are likely to impact food preference and choice. To investigate the impact of genetic variation on food preference, a number of taste phenotypes will be assessed including the inherited ability to taste 6 - $n$-propylthiouracil (PROP). PROP is a bitter thiourea compound; tasters have a greater number fungiform papillae and taste buds on these papillae, making them more sensitive to basic tastes, including fat. ${ }^{23}$ In contrast, non-tasters tend to show heightened acceptance for certain high-fat foods, ${ }^{23}$ and several studies have demonstrated higher BMIs in nontasters compared to taster counterparts. A simple taste test will be used to assess taster/non-taster phenotype status; a salivary DNA sample will also be collected to determine taste receptor genotype(s).

\section{Phase 2 procedures (50 Caucasian, 50 Māori children)}

The protocol for phase 2 is depicted in figure 2 . Cardiovascular health, fatness and fitness measurements (table 1) will take place on the same day in a laboratory setting. Participants will report for testing between the hours of 9:00 and 12:00, having fasted for at least $3 \mathrm{~h}$, and having refrained from exercise for $24 \mathrm{~h} .{ }^{9}$ At the conclusion of the laboratory visit, participants will be provided with a food diary, sleep diary and an accelerometer to collect objective physical activity and sleep data for 7 days. On day 7 , the participant will be asked to complete a web-based questionnaire (described above) in consultation with a parent or guardian.

\section{Cardiovascular disease risk}

Assessments will include flow-mediated dilation (FMD), heart rate variability (HRV) and PWA (as described previously). FMD is the standard tool used to assess endothelial function. ${ }^{24}{ }^{25}$ Reduced FMD is an early marker of atherosclerosis $^{25}$ and an impaired FMD response has been demonstrated in children as young as 7 years old with familial hypercholesterolemia. ${ }^{1}$ FMD will be conducted using a high-resolution B-mode ultrasound device (t3200; Terason, Burlington, Massachusetts, USA) equipped with a $15-4 \mathrm{MHz}$ linear array transducer
(15L4). The raw FMD score (\%) as well as the hyperaemic response (shear stimulus) will be reported. ${ }^{24}$

HRV is a quantitative marker of autonomic activity which has been shown to predict adverse cardiac events and mortality in patients ${ }^{26}$ as well as in the general population. ${ }^{27}$ The $\mathrm{R}$ to $\mathrm{R}$ wave interval will be recorded (Polar RS800CX, Polar Electro OY, Kempele, Finland) during 5 min quiet, supine rest. HRVwill be expressed as the root mean square of successive differences (RMSSD). The z-scores of FMD\%, RMSSD, cSBP and $\mathrm{AIx} \%$ will be summed to create a CVD risk score.

\section{Fatness}

Visceral adiposity poses a higher risk for developing obesity-related disorders than overall adiposity, ${ }^{28}$ including in adolescents and children. ${ }^{29} \mathrm{CT}$ and MRI are reference methods, but both are high-cost technologies, and CT requires radiation exposure. Therefore, intraabdominal tissue thickness (IAT) and abdominal wall fat index (AFI) will be measured by a highly trained sonographist using a portable, high-resolution, B-mode ultrasound device (t3200; Terason, Burlington,Massachusetts, USA) equipped with $6-1 \mathrm{MHz}$ curved array (6C1) and 15-4 MHz linear array (15L4) transducers. IAT will be defined as the distance, in millimetres, between the linea alba and the anterior wall of the aorta, measured $1-5 \mathrm{~cm}$ above the umbilicus at the xipho-umbilical line. AFI is defined as the ratio of subcutaneous to preperitoneal fat, measured immediately below the xiphoid process. Preperitoneal fat is defined as the distance between the linea alba and the peritoneum. These measurements strongly agree with CT-based ${ }^{30}$ and MRI-based ${ }^{31}$ estimations and are highly reliable. ${ }^{31} 32$ The z-scores of IAT and AFI will be summed to create a fatness score.

\section{Physical fitness}

Participants will complete a continuous, incremental maximal exercise test on a cycle ergometer. The test will begin at $10 \mathrm{~W}$ and will increase by $10 \mathrm{~W}$ every minute for the duration of the test. Participants will maintain a cycle cadence of $60 \mathrm{rpm}$ throughout the test. The following criteria will be used to determine test termination: volitional exhaustion in conjunction with a respiratory exchange ratio (RER) $\geq 1.02$, a perception of exertion of 9 or 10 using the Eston-Parfitt Curvilinear scale ${ }^{33}$ and heart rate $\geq 195 \mathrm{bpm}$. As a plateau oxygen uptake is rarely evident in young children, ${ }^{34} \mathrm{VO}_{2}$ peak will also be used to identify maximal oxygen consumption during the test. $\mathrm{VO}_{2}$ peak will be expressed relative to body weight.

\section{Physical activity}

Accelerometry will be used to objectively measure MVPA (min/day). Data collection will take place over 7 days. The participants will be instructed to wear a water resistant 3-axis accelerometer (wActiSleep-BT; ActiGraph LLC, Fort Walton Beach,Florida, USA) on the nondominant arm continuously, that is, $24 \mathrm{~h} /$ day. This is a 
validated objective measure of physical activity for use with young people. ${ }^{35}$

\section{Sleep}

Sleep behaviour will be assessed using the ActiSleep monitor described above in conjunction with a paperbased sleep diary. The monitor will be initialised to collect data across $60 \mathrm{~s}$ epochs. Participants will be instructed to keep a record of time in bed, time out of bed for each measured sleep episode, and to return a completed sleep log diary with the ActiSleep monitor to the research staff. The actigraphy and diary data will be used to determine bedtime and rise time, sleep onset and offset, sleep latency, total sleep time, number and duration of awakenings, wake after sleep onset and sleep efficiency after applying the Sadeh algorithm setting. An integrated ambient light sensor provides information to assist in determining bedtimes and rise times.

\section{Nutrition}

Food intake will be recorded using a 4-day estimated food diary specifically designed for use in this age group. Children will be asked to record everything that they eat and drink (including on one weekend day) with assistance from parents where necessary. Nutrient values will be calculated using the Kai-culator nutrient analysis software (University of Otago 2014) for all macronutrients and key micronutrients of interest.

\section{Sample size calculations}

Phase 1: Using magnitude-based inferences to estimate the sample size required to detect the smallest beneficial (or detrimental) effect in a cross-sectional study (in this case a correlation of 0.10 which is set lower than the estimated correlation of 0.20 found in previous research between fitness and fatness, ${ }^{36}$ with the maximum chances of a type 1 and 2 error set at $5 \%$ (ie, very unlikely), approximately 272 children are required. ${ }^{37}$ To allow for an approximate $30 \%$ non-completion rate across all study measures, 400 children will be recruited to the study.

Phase 2: Using the same method described above, but changing the smallest beneficial effect to match that found previously between FMD and physical activity (ie, $\mathrm{r}=0.40),{ }^{38}$ approximately 20 participants are required per group (Caucasian or Māori). We will oversample to accommodate for setbacks.

\section{Statistical analysis}

All variables will be checked for normality of distribution before analyses and transformations will be applied where necessary. For research Q1 the dependent variable will be fatness (BMI and FMI) and the independent/predictor variables will be physical activity, physical fitness, sleep behaviour and nutrition. Initially, partial correlations will be used to examine bivariate correlations of the predictor variables with BMI, for example. Subsequently, two separate multiple regression models will be used to examine the association of the predictor variables with BMI. Model 1 will include each independent variable separately. Model 2 will adjust for the other predictor variables to test independent associations. The same two models will be used in logistic regression to examine the association of each predictor variable with risk of obesity.

For research $\mathrm{Q}^{2}$ the dependent variable will be physical activity and the predictor variables include walkability index, playability index and the five dimensions of the CAPA scale. Statistical procedures will be similar to those followed for Q1. For research Q3 the dependent variables will be the principal component analysis derived dietary patterns and the independent variables will include food environment variables, psychosocial variables (preferences for and attitudes towards healthy foods) and taste response (taste/non-taste) to PROP. A two-way (environment index $\times$ taste response) analysis of covariance will be used to examine environment-gene interaction, adjusting for psychosocial variables. For research Q4 the dependent variable will be composite cardiometabolic risk score and the predictor variables will include fatness, physical activity, physical fitness, nutrition and sleep behaviour.

For research Q5 the dependent variable will be the composite CVD risk score, and the predictor variables will be fatness, physical fitness, physical activity, sleep and nutrition. Statistical procedures will be similar to those followed for Q1, with the addition of a dichotomous variable to distinguish ethnicity groups (European-descent, Māori). Post hoc, Māori children will be matched to European descent children for BMI. Analysis of covariance will be used to compare groups, adjusting for the other predictor variables.

\section{Dissemination of findings}

Outcomes will be shared with public health professionals and the general public through outlets such as Nutrition Society newsletters and traditional media outlets, including popular newspapers, magazines, radio and television, including ONE Te Karere. In addition, findings will be shared with the academic community through high impact, open access journal articles and through international conferences. Most importantly, results from the study will be disseminated to participating schools and relevant Māori health entities. This will be conducted in a culturally appropriate manner and will be supported and guided by the Māori advisory team. This is likely to include a hui (Māori assembly) where cultural processes are adhered to and which promotes discussion format with participants across all ages and facets of the community, and held at an appropriate venue (ideally a Marae).

\section{DISCUSSION}

Worldwide, health systems are struggling under the escalating burden of non-communicable diseases, of 
which obesity and subsequent CVD are arguably at the forefront. Obesity, and comorbid complications, are occurring at an increasingly younger age, prolonging the burden of disease and resulting in lost years of productivity, a decreased quality of life, and a growing economic burden. The research outcomes from this study will help inform health professionals as to the most effective way to make an impact on the deteriorating health of our children. The stated research will increase the understanding of current diseases affecting Māori children as well as all New Zealand children, and subsequently through more targeted interventions, enhance the health and well-being of children and consequently adults.

Short-term, the findings from this study will elucidate targets for decreasing obesity and improving cardiometabolic health among preadolescent children in New Zealand. The aim is to ensure an immediate impact by disseminating these findings in an applicable manner via popular media, community outlets and traditional academic forums. Long-term, this research will facilitate larger scale prospective and interventional studies, focusing on potential ethnic differences, changes with/across age groups, comparisons between nations and implications on healthcare systems.

\section{Author affiliations \\ ${ }^{1}$ School of Sport and Exercise, Massey University, Wellington, New Zealand \\ ${ }^{2}$ Department of Human Nutrition, University of Otago, Dunedin, New Zealand \\ ${ }^{3}$ Department of Epidemiology, Harvard School of Public Health, Boston, \\ Massachusetts, USA \\ ${ }^{4}$ Institute of Food Nutrition and Human Health, Massey University, Wellington, New Zealand \\ ${ }^{5}$ Sleep/Wake Research Centre, Massey University, Wellington, New Zealand \\ ${ }^{6}$ Department of Environment, Society, and Design, Lincoln University, Christchurch, New Zealand \\ ${ }^{7}$ Faculty of Health, Education and Well-being, University of Wolverhampton, Wolverhampton, UK \\ ${ }^{8}$ Research Centre for Maori Health and Development, Massey University, Wellington, New Zealand}

Contributors Each author: (1) made substantial contributions to conception and design; (2) assisted in drafting the article and provided important intellectual content and (3) gave final approval of the version to be published. A multi-disciplinary approach is required to tackle the proposed research, and as such specific contributions were made by each author, specifically: NC drafted the study methods and will be the project manager of this research study. LS expertise lies in the development and interpretation of non-invasive methodologies for assessing vascular health. LS assisted with the general study design, and describing cardiovascular outcomes. JF assisted with the general study design and with designing the methods for the maximal oxygen consumption test. SL assisted with the general study design. MW was involved in extensive experience in carrying out large-scale multidisciplinary international research. MW specifically assisted with the general study design, biostatistics and genetic study methods. PS expertise lies in food choice and activity, how this relates to risk of chronic conditions, and the role of the physical environment in food choice and activity. PS assisted with nutritional aspect of the study design, and the designing of the electronic questionnaire. DML assisted with the general study design and with designing the methods for the maximal oxygen consumption test. LS assisted with selecting a sleep/ activity monitor device and with the study design to collect and analyse physical activity levels and sleep behaviour data. MT specifically assisted with assessing taste phenotypes and the impact of genetic variation on food preference and choice. TKK expertise lies in health and social outcomes and Māori mental health. TKK specifically assisted with designing study procedures while incorporating Māori culture guidelines. MH assisted with the general study design and with the protocol in the school to measure physical activity levels using the shuttle run test. AML assisted with describing well-being outcomes and psychosocial measuring methods. DM specifically assisted with selecting a sleep/activity monitor device and with the study design to collect and analyse physical activity levels and sleep behaviour data.

Funding Seed funding was provided by Massey University Research Fund.

Ethic approval Ethical approval has been obtained from the regional Health and Disabilities Ethics Committee (14/CEN/83).

Competing interests None.

Provenance and peer review Not commissioned; externally peer reviewed.

Data sharing statement No additional data are available.

Open Access This is an Open Access article distributed in accordance with the Creative Commons Attribution Non Commercial (CC BY-NC 4.0) license, which permits others to distribute, remix, adapt, build upon this work noncommercially, and license their derivative works on different terms, provided the original work is properly cited and the use is non-commercial. See: http:// creativecommons.org/licenses/by-nc/4.0/

\section{REFERENCES}

1. Sorensen KE, Celermajer DS, Georgakopoulos D, et al. Impairment of endothelium-dependent dilation is an early event in children with familial hypercholesterolemia and is related to the lipoprotein(a) level. J Clin Invest 1994;93:50-5.

2. Lambrick DM, Stoner L, Faulkner J, et al. Preventive medicine needs to begin with our children. Int J Prev Med 2014;5:129-31.

3. Olshansky SJ, Passaro DJ, Hershow RC, et al. A potential decline in life expectancy in the United States in the 21st century. $N$ Engl $J$ Med 2005;352:1138-45.

4. MoH. New Zealand health survey: annual update of key findings 2012/13. Wellington: Ministry of Health, 2014.

5. Stoner L, Stoner KR, Young JM, et al. Preventing a cardiovascular disease epidemic among indigenous populations through lifestyle changes. Int J Prev Med 2012;3:230-40.

6. Hill JO, Wyatt HR, Reed GW, et al. Obesity and the environment: where do we go from here? Science 2003;299:853-5.

7. Waters E, de Silva-Sanigorski A, Hall BJ, et al. Interventions for preventing obesity in children. Cochrane Database Syst Rev 2011; (12):CD001871.

8. Carter PJ, Taylor BJ, Williams SM, et al. Longitudinal analysis of sleep in relation to BMI and body fat in children: the FLAME study. BMJ 2011;342:d2712.

9. Stoner L, Lambrick DM, Faulkner J, et al. Guidelines for the use of pulse wave analysis in adults and children. J Atheroscler Thromb 2013;20:404-6.

10. Vlachopoulos C, Aznaouridis K, O'Rourke MF, et al. Prediction of cardiovascular events and all-cause mortality with central haemodynamics: a systematic review and meta-analysis. Eur Heart J 2010;31:1865-71.

11. Stoner L, Lambrick DM, Westrupp N, et al. Validation of oscillometric pulse wave analysis measurements in children. Am J Hypertens 2014;27:865-72.

12. Cole TJ, Bellizzi MC, Flegal KM, et al. Establishing a standard definition for child overweight and obesity worldwide: international survey. BMJ 2000;320:1240-3.

13. Melo X, Santa-Clara H, Almeida J, et al. Comparing several equations that predict peak VO2 using the 20-m multistage-shuttle run-test in 8-10-year-old children. Eur J Appl Physiol 2011;111:839-49.

14. Wong JE, Parnell W, Black KE, et al. Reliability and relative validity of a food frequency questionnaire to assess food group intakes in New Zealand adolescents. Nutr J 2012;11:65

15. Chen X, Beydoun MA, Wang Y. Is sleep duration associated with childhood obesity? A systematic review and meta-analysis. Obesity (Silver Spring) 2008;16:265-74.

16. Owens JA, Spirito A, McGuinn M, et al. Sleep habits and sleep disturbance in elementary school-aged children. J Dev Behav Pediatr 2000;21:27-36.

17. Badland HM, Schofield GM, Witten $\mathrm{K}$, et al. Understanding the Relationship between Activity and Neighbourhoods (URBAN) Study: research design and methodology. BMC Public Health 2009;9:224.

18. Frank LD, Saelens BE, Chapman J, et al. Objective assessment of obesogenic environments in youth: geographic information system 
methods and spatial findings from the Neighborhood Impact on Kids study. Am J Prev Med 2012;42:e47-55.

19. Brustad RJ. Parental and psychological influences on children's attraction to physical activity. Pediatr Exerc Sci 1993:5:210-23.

20. Rose E, Larkin D, Hands B, et al. Evidence for the validity of the children's attraction to physical activity questionnaire (CAPA) with young children. J Sci Med Sport 2009;12:573-8.

21. Sandvik C, De Bourdeaudhuij I, Due P, et al. Personal, social and environmental factors regarding fruit and vegetable intake among schoolchildren in nine European countries. Ann Nutr Metab 2005;49:255-66.

22. Mainland JD, Keller A, Li YR, et al. The missense of smell: functional variability in the human odorant receptor repertoire. Nat Neurosci 2014;17:114-20.

23. Tepper BJ. PROP taster status is related to fat perception and preference. Ann NY Acad Sci Am 1998;30:802-4.

24. Stoner L, Sabatier MJ. Use of ultrasound for non-invasive assessment of flow-mediated dilation. $J$ Atheroscler Thromb 2012;19:407-21.

25. Celermajer DS, Sorensen KE, Gooch VM, et al. Non-invasive detection of endothelial dysfunction in children and adults at risk of atherosclerosis. Lancet 1992;340:1111-15.

26. La Rovere MT, Pinna GD, Maestri R, et al. Short-term heart rate variability strongly predicts sudden cardiac death in chronic heart failure patients. Circulation 2003;107:565-70.

27. Tsuji H, Larson MG, Venditti FJ Jr, et al. Impact of reduced heart rate variability on risk for cardiac events. The Framingham Heart Study. Circulation 1996:94:2850-5.

28. Fox CS, Massaro JM, Hoffmann U, et al. Abdominal visceral and subcutaneous adipose tissue compartments: association with metabolic risk factors in the Framingham Heart Study. Circulation 2007;116:39-48.
29. Owens S, Gutin B, Ferguson M, et al. Visceral adipose tissue and cardiovascular risk factors in obese children. $J$ Pediatr 1998;133:41-5.

30. Leite CC, Wajchenberg BL, Radominski R, et al. Intra-abdominal thickness by ultrasonography to predict risk factors for cardiovascular disease and its correlation with anthropometric measurements. Metabolism 2002;51:1034-40.

31. Stolk RP, Wink O, Zelissen PM, et al. Validity and reproducibility of ultrasonography for the measurement of intra-abdominal adipose tissue. Int J Obes Relat Metab Disord 2001;25:1346-51.

32. Bazzocchi A, Filonzi G, Ponti F, et al. Accuracy, reproducibility and repeatability of ultrasonography in the assessment of abdominal adiposity. Acad Radiol 2011;18:1133-43.

33. Eston RG, Lambrick DM, Rowlands AV. The perceptual response to exercise of progressively increasing intensity in children aged 7-8 years: validation of a pictorial curvilinear ratings of perceived exertion scale. Psychophysiology 2009;46:843-51.

34. Stratton G, Williams C, eds. Children and Fitness Testing. Sports and exercise physiology testing guidelines: The British association of sport and exercise sciences quide. Routledge, Oxon, 2007:321-33.

35. Pate RR, O'Neill JR, Mitchell J. Measurement of physical activity in preschool children. Med Sci Sports Exerc 2010;42:508-12.

36. Moschonis G, Mougios V, Papandreou C, et al. "Leaner and less fit" children have a better cardiometabolic profile than their "heavier and more fit" peers: The Healthy Growth Study. Nutr Metab Cardiovasc Dis 2013;23:1058-65.

37. Hopkins WG, Marshall SW, Batterham AM, et al. Progressive statistics for studies in sports medicine and exercise science. Med Sci Sports Exerc 2009;41:3-13.

38. Abbott RA, Harkness MA, Davies PS. Correlation of habitual physical activity levels with flow-mediated dilation of the brachial artery in 5-10 year old children. Atherosclerosis 2002;160:233-9. 